\title{
MEAN CONVERGENCE OF GENERALIZED WALSH-FOURIER SERIES
}

BY

\author{
WO-SANG YOUNG
}

\begin{abstract}
Paley proved that Walsh-Fourier series converges in $L^{p}$ $(1<p<\infty)$. We generalize Paley's result to Fourier series with respect to characters of countable direct products of finite cyclic groups of arbitrary orders.
\end{abstract}

1. Introduction. It is known that the Walsh functions are characters of the countable direct product of groups of order 2 . In this note we consider characters of $\Pi_{i=0}^{\infty} Z_{p_{i}}$, where $Z_{p_{i}}$ is a cyclic group of order $p_{i}, p_{i} \geqslant 2$. Various Fourier properties of this generalized Walsh system have been studied in [8], [7], [9], $[5],[3],[4],[2]$, and others. Many of these results are obtained only for the case where $\sup _{i} p_{i}<\infty$. In fact, Price [7] showed that some basic properties no longer hold when $\sup _{i} p_{i}=\infty$. We will show that results concerning mean convergence, however, are still valid even if the orders $p_{i}$ are unbounded. The bounded case was first obtained by Watari [9]. See also Gosselin [2].

The author would like to thank Richard Hunt and Charles Fefferman for several helpful conversations.

Let $\left\{p_{i}\right\}_{i \geqslant 0}$ be a sequence of integers, $p_{i} \geqslant 2$. Let $G=\Pi_{i=0}^{\infty} Z_{p_{i}}$ be the direct product of cyclic groups of order $p_{i}$, and $\mu$ the Haar measure on $G$ normalized by $\mu(G)=1$. Each element of $G$ can be considered as a sequence $\left\{x_{i}\right\}$, with $0 \leqslant x_{i}<p_{i}$. Set $m_{0}=1, m_{k}=\Pi_{i=0}^{k-1} p_{i}, k=1,2, \ldots$. We can identify $G$ with the unit interval $(0,1)$. This identification consists in associating with each $\left\{x_{i}\right\}$ $\in G, 0 \leqslant x_{i}<p_{i}$, the point $\Sigma_{i=0}^{\infty} x_{i} m_{i+1}^{-1} \in(0,1)$. If we disregard the countable set of $p_{i}$-rationals, this mapping is one-one, onto and measure preserving.

We define an orthonormal system of functions $\left\{\phi_{k}\right\}$ on $G$. For each $x=$ $\left\{x_{i}\right\} \in G$, let $\phi_{k}(x)=\exp \left(2 \pi i x_{k} / p_{k}\right), k=0,1, \ldots$. We enumerate the set of all finite products of $\left\{\phi_{k}\right\}$ using a scheme of Paley. We express each nonnegative integer $n$ as a finite sum $n=\Sigma_{k=0}^{\infty} \alpha_{k} m_{k}$, with $0 \leqslant \alpha_{k}<p_{k}$, and define $\chi_{n}=$ $\Pi_{k=0}^{\infty} \phi_{k}^{\alpha_{k}}$. The functions $\left\{\chi_{n}\right\}$ are the characters of $G$, and they form a complete orthonormal system on $G$. For the case $p_{i}=2, i=0,1, \ldots, G$ is the dyadic group, $\left\{\phi_{k}\right\}$ are the Rademacher functions, and $\left\{\chi_{n}\right\}$ the Walsh functions.

Presented to the Society, April 11, 1975; received by the editors July 30, 1974 and, in revised form, February 19, 1975.

AMS (MOS) subject classifications (1970). Primary 42A20, 42A40, 42A56, 43A50. 
We consider Fourier series with respect to $\left\{\chi_{n}\right\}$. Let $D_{n}=\sum_{j=0}^{n-1} \chi_{j}, n=$ $1,2, \ldots$, be the $n$th Dirichlet kernel. For $f \in L^{1}(G)$,

$$
S_{n} f(x)=\int_{G} f(t) D_{n}(x-t) d \mu(t), \quad n=1,2, \ldots,
$$

denotes the $n$th partial sum of the Fourier series of $f$. We have the following uniform estimates on $\left\{S_{n} f\right\}$.

THEOREM 1. There are absolute constants $C$ and $C_{p}$ such that, for $n=$ $1,2, \ldots$,

$$
\begin{aligned}
& \left\|S_{n} f\right\|_{p} \leqslant C_{p}\|f\|_{p}, \quad f \in L^{p}(G), 1<p<\infty, \\
& \mu\left\{\left|S_{n} f\right|>y\right\} \leqslant C y^{-1}\|f\|_{1}, \quad f \in L^{1}(G), y>0 .
\end{aligned}
$$

These results and the density of the generalized Walsh polynomials imply the mean convergence of $S_{n} f$ to $f$ in $L^{p}(G), 1<p<\infty$.

The constants $C$ and $C_{p}$ in the above theorem are independent of the orders $p_{i}$ of the cyclic groups.

If $p_{i}=2, i=0,1, \ldots$, Theorem 1 is Paley's result for the Walsh-Fourier series [6]. On the other hand, if $p_{0} \rightarrow \infty, S_{n} f$ resembles the $n$th trigonometric partial sum. Thus, when restricted to one cyclic group, Theorem 1 can be viewed as a discrete analogue of M. Riesz's theorem for the trigonometric Fourier series [10, I, p. 266].

In what follows $C$ will denote an absolute constant, which may vary from line to line.

2. Modified partial sums and conjugate functions. We will use the following notation. Let $\left\{G_{k}\right\}$ be a sequence of subgroups of $G$ defined by

$$
G_{0}=G, \quad G_{k}=\prod_{i=0}^{k-1}\{0\} \times \prod_{i=k}^{\infty} Z_{p_{i}}, \quad k=1,2, \ldots
$$

Then $\mu\left(G_{k}\right)=m_{k}^{-1}$. Let $F_{k}$ be the $\sigma$-algebra generated by the cosets of $G_{k}$. On the interval $(0,1)$, atoms of $F_{k}$ are intervals of the form $\left(j m_{k}^{-1},(j+1) m_{k}^{-1}\right)$, $j=0,1, \ldots, m_{k}-1$. We note that $\phi_{k}$ is measurable with respect to $F_{k+1}$.

It is proved in [8] that

$$
D_{m_{k}}(x)= \begin{cases}m_{k} & \text { if } x \in G_{k} \\ 0 & \text { otherwise }\end{cases}
$$

From (3) it follows that

$$
S_{m_{k}} f(x)=\frac{1}{\mu(I)} \int_{I} f d \mu,
$$

where $I=x+G_{k}$. 
It is also proved in [8] that if $n=\Sigma_{k=0}^{\infty} \alpha_{k} m_{k}, 0 \leqslant \alpha_{k}<p_{k}$,

$$
D_{n}=\chi_{n} \sum_{k=0}^{\infty} D_{m_{k}} \phi_{k}^{-\alpha_{k}}\left(\sum_{j=0}^{\alpha_{k}-1} \phi_{k}^{j}\right) \text {. }
$$

with the interpretation that $\Sigma_{j=0}^{\alpha_{k}-1} \phi_{k}^{j}=0$ if $\alpha_{k}=0$. It is convenient to consider the modified Dirichlet kernel $D_{n}^{*}$ defined by $D_{n}^{*}=\bar{\chi}_{n} D_{n}$. From (4) we have

$$
D_{\alpha_{k} m_{k}}^{*}=D_{m_{k}} \phi_{k}^{-\alpha_{k}}\left(\sum_{j=0}^{\alpha_{k}-1} \phi_{k}^{j}\right)=D_{m_{k+1}}-D_{\left(p_{k}-\alpha_{k}\right) m_{k}},
$$

and

$$
D_{n}^{*}=\sum_{k=0}^{\infty} D_{\alpha_{k} m_{k}}^{*}
$$

Let $S_{n}^{*} f(x)=\int_{G} f(t) D_{n}^{*}(x-t) d \mu(t)$ be the $n$th modified partial sum. Since $S_{n}^{*} f=\bar{\chi}_{n} S_{n}\left(f \chi_{n}\right)$, Theorem 1 is equivalent to

THEOREM 1*. There are absolute constants $C$ and $C_{p}$ such that, for $n=$ $1,2, \ldots$,

$$
\begin{aligned}
& \left\|S_{n}^{*} f\right\|_{p} \leqslant C_{p}\|f\|_{p}, \quad f \in L^{p}(G), 1<p<\infty, \\
& \mu\left\{\left|S_{n}^{*} f\right|>y\right\} \leqslant C y^{-1}\|f\|_{1}, \quad f \in L^{1}(G), y>0 .
\end{aligned}
$$

We will prove Theorem $1^{*}$. The following facts concerning the modified partial sums will be needed. First of all we have, by (5) and (6),

$$
S_{n}^{*} f=\sum_{k=0}^{\infty} S_{\alpha_{k} m_{k}}^{*} f
$$

with $S_{\alpha_{k} m_{k}}^{*} f=S_{m_{k+1}} f-S_{\left(p_{k}-\alpha_{k}\right) m_{k}} f$. Moreover, it follows from (5) and (3) that

$$
S_{\alpha_{k} m_{k}}^{*} f(x)=\frac{1}{\mu(I)} \int_{I} f(t) \phi_{k}^{-\alpha_{k}}(x-t)\left(\sum_{j=0}^{\alpha^{-1}} \phi_{k}^{j}(x-t)\right) d \mu(t),
$$

where $I=x+G_{k}$. Now, for $f \in L^{1}(G)$,

$$
\frac{1}{\mu(I)} \int_{I} f(t)\left(\sum_{j=0}^{\alpha^{-1}} \phi_{k}^{j}(x-t)\right) d \mu(t)
$$

resembles the $\alpha_{k}$ th partial sum of the trigonometric Fourier series of $f$ on the coset $I$. The relation between the trigonometric partial sum and conjugate function leads to our definition of the conjugate function $H_{k} f$ of $f \in L^{1}(G)$. Let $x=\left\{x_{k}\right\} \in G$. We define

$$
H_{k} f(x)=\frac{1}{2} \frac{1}{\mu(I)} \int_{I \cap\left\{x_{k} \neq t_{k}\right\}} f(t) \cot \left(\pi\left(x_{k}-t_{k}\right) / p_{k}\right) d \mu(t),
$$


where $I=x+G_{k}$. Since

$$
\phi_{k}^{-\alpha}(t) \sum_{j=0}^{\alpha_{k}-1} \phi_{k}^{j}(t)=\left\{\begin{array}{l}
\alpha_{k} \text { if } t_{k}=0, \\
1 / 2 \phi_{k}^{-\alpha_{k}}(t)-1 / 2+1 / 2 i \phi_{k}^{-\alpha_{k}}(t) \cot \left(\pi t_{k} / p_{k}\right) \\
-1 / 2 i \cot \left(\pi t_{k} / p_{k}\right) \text { if } t_{k} \neq 0
\end{array}\right.
$$

(10) implies

$$
\begin{aligned}
S_{\alpha_{k} m_{k}}^{*} f(x)= & \frac{\alpha_{k}}{\mu(I)} \int_{I \cap\left\{x_{k}=t_{k}\right\}} f(t) d \mu(t) \\
& +\frac{1}{2} \phi_{k}^{-\alpha_{k}}(x) \frac{1}{\mu(I)} \int_{I \cap\left\{x_{k} \neq t_{k}\right\}} f(t) \phi_{k}^{\alpha_{k}}(t) d \mu(t) \\
& -\frac{1}{2} \frac{1}{\mu(I)} \int_{I \cap\left\{x_{k} \neq t_{k}\right\}} f(t) d \mu(t) \\
& +i \phi_{k}^{-\alpha}(x) H_{k}\left(f \phi_{k}^{\alpha_{k}}\right)(x)-i H_{k} f(x) .
\end{aligned}
$$

(9) and (11) will be used later in the proof of Theorem $1^{*}$.

3. A decomposition lemma. For the proof of Theorem $1^{*}$ we need a modified form of the Calderón-Zygmund decomposition lemma [1, p. 91]. The following may best be described on the interval $(0,1)$.

LEMMA 2. Let $f$ belong to $L^{1}(G)$ and $y>0$ with $\|f\|_{1} \leqslant y$. Let $\left\{\alpha_{k}\right\}_{k \geqslant 0}$ be a sequence of integers with $0 \leqslant \alpha_{k}<p_{k}$. Then there are $L^{1}$ functions $g$ and $b$, and a collection $C=\left\{\omega_{j}\right\}$ of disjoint intervals such that

(12) $f=g+b$.

(13) $|g| \leqslant C y$ a.e.

(14) $\|g\|_{1} \leqslant C\|f\|_{1}$.

(15) $C=\bigcup_{k=0}^{\infty} C_{k}$ where each $\omega_{j} \in C_{k}$ is measurable with respect to $F_{k+1}$ and is a proper subset of a coset of $G_{k}$.

(16) $b(x)=0$ if $x \notin \bigcup_{j} \omega_{j}$.

(17) $\int_{\omega_{j}} b d \mu=0$ for every $\omega_{j} \in C$ and $\int_{\omega_{j}} b \phi_{k}^{\alpha} k d \mu=0$ for every $\omega_{j} \in C_{k}$, $k=0,1, \ldots$.

(18) $\int_{\omega_{j}}|b| d \mu \leqslant C \int_{\omega_{j}}|f| d \mu$ for every $\omega_{j} \in C$.

(19) $\Sigma_{j} \mu\left(\omega_{j}\right) \leqslant y^{-1}\|f\|_{1}$.

Proof. We first construct the collection $C$ of disjoint intervals. We divide $(0,1)$ into two subintervals $I_{1}$ and $I_{1}^{\prime}$, with $I_{1}, I_{1}^{\prime} \in F_{1}$ and $\mu\left(I_{1}\right)-m_{1}^{-1} \leqslant \mu\left(I_{1}^{\prime}\right)$ $\leqslant \mu\left(I_{1}\right)$. If $\left(1 / \mu\left(I_{1}\right)\right) \int_{I_{1}}|f| d \mu>y$, then $I_{1}$ is in $C$. Otherwise we repeat the above process with $(0,1)$ replaced by $I_{1}$. We do the same with $I_{1}^{\prime}$. Finally we reach a stage where the subinterval $I$ is an atom of $F_{1}$ and $(1 / \mu(I)) \int_{I}|f| d \mu \leqslant y$. 
We then divide $I$ into subintervals $I_{2}$ and $I_{2}^{\prime}$, with $I_{2}, I_{2}^{\prime} \in F_{2}$ and $\mu\left(I_{2}\right)-m_{2}^{-1} \leqslant$ $\mu\left(I_{2}^{\prime}\right) \leqslant \mu\left(I_{2}\right)$, and proceed as before. In this way we obtain a collection $C=$ $\left\{\omega_{j}\right\}$ of disjoint intervals which has the properties that

$$
y<\frac{1}{\mu\left(\omega_{j}\right)} \int_{\omega_{j}}|f| d \mu \leqslant 3 y, \quad \omega_{j} \in C,
$$

and

$$
|f(x)| \leqslant y \quad \text { for a.e. } x \notin \bigcup_{j} \omega_{j} .
$$

The first inequality of (20) implies (19). Set

$$
C_{0}=\left\{\omega_{j} \in C: \omega_{j} \in F_{1}\right\}
$$

and

$$
C_{k}=\left\{\omega_{j} \in C \backslash \bigcup_{l=0}^{k-1} C_{l}: \omega_{j} \in F_{k+1}\right\}
$$

$k=1,2, \ldots$ Then $\left\{C_{k}\right\}$ satisfies (15).

Next we decompose $f$ as $f=g \neq b$, with

$$
g(x)=\left\{\begin{array}{l}
f(x) \quad \text { if } x \notin \bigcup_{j} \omega_{j}, \\
a_{k j}+b_{k j} \phi_{k}^{-\alpha}(x) \text { if } x \in \omega_{j} \in C_{k},
\end{array}\right.
$$

where $a_{k j}, b_{k j}$ are constants chosen in such a way that

$$
\int_{\omega_{j}} f d \mu=\int_{\omega_{j}}\left(a_{k j}+b_{k j} \phi_{k}^{-\alpha}\right) d \mu
$$

and

$$
\int_{\omega_{j}} f \phi_{k}^{\alpha_{k}} d \mu=\int_{\omega_{j}}\left(a_{k j}+b_{k j} \phi_{k}^{-\alpha_{k}}\right) \phi_{k}^{\alpha_{k}} d \mu .
$$

Then $b=g-f$ automatically satisfies (16) and (17). The proof will be completed if we show

$$
|g(x)| \leqslant \frac{C}{\mu\left(\omega_{j}\right)} \int_{\omega_{j}}|f| d \mu, \quad x \in \omega_{j}, \omega_{j} \in C,
$$

for then (25) together with (20) and (21) will imply (13), (14) and (18).

To prove (25) we write $\beta_{k}=\alpha_{k}$ if $0 \leqslant \alpha_{k} \leqslant p_{k} / 2$ and $\beta_{k}=\alpha_{k}-p_{k}$ if $p_{k} / 2<\alpha_{k}<p_{k}$. Then $-p_{k} / 2<\beta_{k} \leqslant p_{k} / 2$ and $\phi_{k}^{\alpha_{k}}=\phi_{k}^{\beta_{k}}$. Let $\omega_{j} \in C_{k}$. If $\omega_{j}$ is a coset of $G_{k+1}$, or if $\beta_{k}=0$, then $\phi_{k}$ is constant in $\omega_{j}$. In this case we set $a_{k j}=\left(\mu\left(\omega_{j}\right)\right)^{-1} \int_{\omega_{j}} f d \mu$ and $b_{k j}=0$. (25) follows immediately.

Now suppose $\beta_{k} \neq 0$ and $\omega_{j}$ is not a coset of $G_{k+1}$, that is $\mu\left(\omega_{j}\right) m_{k+1} \geqslant$ 2. Then $\left|\left(\mu\left(\omega_{j}\right)\right)^{-1} \int_{\omega_{j}} \phi_{k}^{\beta_{k}} d \mu\right| \neq 1$. Solving (23), (24) for $a_{k j}, b_{k j}$ and substituting into (22) we obtain, for $x \in \omega_{j}$, 
316

WO-SANG YOUNG

$$
\begin{aligned}
g(x)= & {\left[\frac{1}{\mu\left(\omega_{j}\right)} \int_{\omega_{j}} f d \mu-\frac{1}{\mu\left(\omega_{j}\right)} \int_{\omega_{j}} \phi_{k}^{-\beta_{k}} d \mu \frac{1}{\mu\left(\omega_{j}\right)} \int_{\omega_{j}} f \phi_{k}^{\beta_{k}} d \mu\right.} \\
& +\frac{1}{\mu\left(\omega_{j}\right)} \int_{\omega_{j}} f \phi_{k}^{\beta_{k}} d \mu \phi_{k}^{-\beta_{k}}(x) \\
& \left.\quad-\frac{1}{\mu\left(\omega_{j}\right)} \int_{\omega_{j}} \phi_{k}^{\beta_{k}} d \mu \frac{1}{\mu\left(\omega_{j}\right)} \int_{\omega_{j}} f d \mu \phi_{k}^{-\beta_{k}}(x)\right] \\
= & {\left[\frac{1}{\mu\left(\omega_{j}\right)} \int_{\omega_{j}} f(y) \frac{1}{\mu\left(\omega_{j}\right)} \int_{\omega_{j}}\left(\phi_{k}^{\beta_{k}}(y)-\phi_{k}^{\beta_{k}}(t)\right)\right.} \\
& \left.\times\left.\left[1-\left|\frac{1}{\mu\left(\omega_{j}\right)} \int_{\omega_{j}} \phi_{k}^{\beta_{k}} d \mu\right|^{2}\right]^{-1} \int_{\omega_{j}} \phi_{k}^{\beta_{k}} d \mu\right|^{2}\right]^{-1} .
\end{aligned}
$$

Observe that for $s, t \in \omega_{j}$,

$$
\begin{aligned}
\left|\phi_{k}^{\beta_{k}}(s)-\phi_{k}^{\beta_{k}}(t)\right| & \leqslant\left|2 \pi \beta_{k} / p_{k}\right|\left|s_{k}-t_{k}\right| \\
& \leqslant\left(2 \pi\left|\beta_{k}\right| / p_{k}\right) \mu\left(\omega_{j}\right) m_{k+1}=2 \pi\left|\beta_{k}\right| \mu\left(\omega_{j}\right) m_{k},
\end{aligned}
$$

and

$$
\left|\phi_{k}^{\beta_{k}}(s)-\phi_{k}^{\beta_{k}}(t)\right| \leqslant 2
$$

Also,

$$
\left|\frac{1}{\mu\left(\omega_{j}\right)} \int_{\omega_{j}} \phi_{k}^{\beta_{k}} d \mu\right|=\left|\frac{1-\exp \left(2 \pi i \beta_{k} \mu\left(\omega_{j}\right) m_{k}\right)}{\mu\left(\omega_{j}\right) m_{k+1}\left(1-\exp \left(2 \pi i \beta_{k} / p_{k}\right)\right)}\right| .
$$

Therefore, for $x \in \omega_{j}$,

$$
\begin{aligned}
|g(x)| \leqslant & \frac{1}{\mu\left(\omega_{j}\right)} \int_{\omega_{j}}|f| d \mu \min \left(4,\left(2 \pi \beta_{k} \mu\left(\omega_{j}\right) m_{k}\right)^{2}\right) \\
& \times\left[1-\left|\frac{1-\exp \left(2 \pi i \beta_{k} \mu\left(\omega_{j}\right) m_{k}\right)}{\mu\left(\omega_{j}\right) m_{k+1}\left(1-\exp \left(2 \pi i \beta_{k} / p_{k}\right)\right)}\right|^{2}\right]^{-1} .
\end{aligned}
$$

A direct calculation shows that for any integer $n \geqslant 2$ and any number $\theta$ with $-\pi<\theta \leqslant \pi$, we have

$$
(n \theta)^{2}\left[1-\left|\left(1-e^{i n \theta}\right) / n\left(1-e^{i \theta}\right)\right|^{2}\right]^{-1} \leqslant C
$$

for $n|\theta| \leqslant \pi / 10$, and 


$$
\left[1-\left|\left(1-e^{i n \theta}\right) / n\left(1-e^{i \theta}\right)\right|^{2}\right]^{-1} \leqslant C
$$

for $n|\theta| \geqslant \pi / 10$. (25) now follows immediately from (26), (27) and (28). This concludes the proof of the lemma.

4. Proof of Theorem $1^{*}$. The case $p=2$ of (7) is a consequence of Plancherel's formula. It therefore suffices to prove (8), for then (7) will follow by the Marcinkiewicz interpolation theorem [10, II, p. 112] and a duality argument.

For the proof of (8) we note that there is nothing to prove if $\|f\|_{1}>y$, so we can assume $\|f\|_{1} \leqslant y$. Decompose $f$ as in Lemma 2. Since

$$
\mu\left\{\left|S_{n}^{*} f\right|>y\right\} \leqslant \mu\left\{\left|S_{n}^{*} g\right|>y / 2\right\}+\mu\left\{\left|S_{n}^{*} b\right|>y / 2\right\},
$$

(8) will follow if we can show that each term on the right is bounded by $C y^{-1}\|f\|_{1}$.

Using the fact that $\left\{S_{n}^{*}\right\}$ is uniformly bounded in $L^{2}$, we obtain

$$
\mu\left\{\left|S_{n}^{*} g\right|>y / 2\right\} \leqslant C y^{-2}\left\|S_{n}^{*} g\right\|_{2}^{2} \leqslant C y^{-2}\|g\|_{2}^{2} \leqslant C y^{-1}\|f\|_{1},
$$

by (13) and (14).

To estimate $\left|S_{n}^{*} b\right|$ we use the following notation. Let $\omega_{j} \in F_{k+1}$, with $\omega_{j}$ contained in the coset $I$ of $G_{k}$. We consider $I$ as a circle, and let $\omega_{j}^{*}$ denote the interval inside $I$ which contains $\omega_{j}$ at its center and $\mu\left(\omega_{j}^{*}\right)=3 \mu\left(\omega_{j}\right)$. Let $\Omega^{*}=\bigcup_{j} \omega_{j}^{*}$. We have, by (19),

$$
\mu\left(\Omega^{*}\right) \leqslant 3 \sum_{j} \mu\left(\omega_{j}\right) \leqslant 3 y^{-1}\|f\|_{1} .
$$

Therefore it suffices to prove

$$
\mu\left\{x \notin \Omega^{*}:\left|S_{n}^{*} b\right|>y / 2\right\} \leqslant C y^{-1}\|f\|_{1} .
$$

To do this we expand $S_{n}^{*} b$ as in (9) and (11). Moreover, we observe that for $x \notin \Omega^{*}$ the first three terms in (11) vanish. This can be seen as follows. Let $I=x+G_{k}$ and $I^{\prime}=x+G_{k+1}$. Then neither $I$ nor $I^{\prime}$ is contained in $\bigcup_{j} \omega_{j}$. For the first term in (11), we have

$$
\int_{I \cap\left\{x_{k}=t_{k}\right\}} b(t) d \mu(t)=\sum_{\omega_{j} \subset r^{\prime}} \int_{\omega_{j}} b d \mu=0,
$$

by (16) and (17). For the second term,

$$
\begin{aligned}
\int_{I \cap\left\{x_{k}=t_{k}\right\}} b(t) \phi_{k}^{\alpha}(t) d \mu(t)= & \sum_{\omega_{j} \subset I ; \omega_{j} \notin I^{\prime}} \int_{\omega_{j}} b(t) \phi_{k}^{\alpha}(t) d \mu(t) \\
= & \sum_{\omega_{j} \subset I ; \omega_{j} \in c_{k}} \int_{\omega_{j}} b(t) \phi_{k}^{\alpha_{k}}(t) d \mu(t) \\
& +\sum_{\omega_{j} \subset I ; \omega_{j} \Phi I^{\prime} ; \omega_{j} \notin c_{k}} \int_{\omega_{j}} b(t) \phi_{k}^{\alpha_{k}}(t) d \mu(t) .
\end{aligned}
$$


If $\omega_{j} \in C_{k}$, then $\int_{\omega_{j}} b \phi_{k}^{\alpha_{k}} d \mu=0$, by (17). If $\omega_{j} \subset I$ and $\omega_{j} \notin C_{k}$, then $\phi_{k}^{\alpha_{k}}$ is constant on $\omega_{j}$, so $\int_{\omega_{j}} b \phi_{k}^{\alpha_{k}} d \mu=0$ by (17). Hence $\int_{I \cap\left\{x_{k} \neq t_{k}\right\}} b(t) \phi_{k}^{\alpha_{k}}(t) d \mu(t)$ $=0$. Similarly $\int_{I \cap\left\{x_{k} \neq t_{k}\right\}} b(t) d \mu(t)=0$. Therefore we have

$$
S_{\alpha_{k} m_{k}}^{*} b(x)=i \phi_{k}^{-\alpha_{k}}(x) H_{k}\left(b \phi_{k}^{\alpha k}\right)(x)-i H_{k} b(x), \quad x \notin \Omega^{*} .
$$

Thus, if $x \notin \Omega^{*}$,

$$
\left|S_{n}^{*} b(x)\right| \leqslant \sum_{k=0}^{\infty}\left|S_{\alpha_{k} m_{k}}^{*} b(x)\right| \leqslant \sum_{k=0}^{\infty}\left|H_{k}\left(b \phi_{k}^{\alpha_{k}}\right)(x)\right|+\sum_{k=0}^{\infty}\left|H_{k} b(x)\right| .
$$

(29) will be proved if we can show

$$
\mu\left\{x \notin \Omega^{*}: \sum_{k=0}^{\infty}\left|H_{k}\left(b \phi_{k}^{\alpha_{k}}\right)(x)\right|>\frac{y}{4}\right\} \leqslant C y^{-1}\|f\|_{1}
$$

and

$$
\mu\left\{x \notin \Omega^{*}: \sum_{k=0}^{\infty}\left|H_{k} b(x)\right|>\frac{y}{4}\right\} \leqslant C y^{-1}\|f\|_{1} .
$$

We will demonstrate (31). (32) can be proved similarly.

Suppose $x \notin \Omega^{*}$. Let $I=x+G_{k}$ and $I^{\prime}=x+G_{k+1}$. Then, as before, we have

$$
\begin{aligned}
H_{k}\left(b \phi_{k}^{\alpha_{k}}\right)(x)= & \frac{1}{2} \frac{1}{\mu(I)} \sum_{\omega_{j} \subset I ; \omega_{j} q I^{\prime}} \int_{\omega_{j}} b(t) \phi_{k}^{\alpha_{k}}(t) \cot \left(\frac{\pi\left(x_{k}-t_{k}\right)}{p_{k}}\right) d \mu(t) \\
= & \frac{1}{2} \frac{1}{\mu(I)} \sum_{\omega_{j} \subset I ; \omega_{j} \in c_{k}} \int_{\omega_{j}} b(t) \phi_{k}^{\alpha_{k}}(t) \cot \left(\frac{\pi\left(x_{k}-t_{k}\right)}{p_{k}}\right) d \mu(t) \\
& +\frac{1}{2} \frac{1}{\mu(I)} \sum_{\omega_{j} \subset I ; \omega_{j} q I^{\prime} ; \omega_{j} \notin c_{k}} \int_{\omega_{j}} b(t) \phi_{k}^{\alpha_{k}}(t) \cot \left(\frac{\pi\left(x_{k}-t_{k}\right)}{p_{k}}\right) d \mu(t) .
\end{aligned}
$$

Again, if $\omega_{j} \subset I$ and $\omega_{j} \notin C_{k}, \phi_{k}^{\alpha_{k}}(t) \cot \left(\pi\left(x_{k}-t_{k}\right) / p_{k}\right)$ is constant on $\omega_{j}$. Therefore the last term on the right vanishes by (17). Moreover, if $\omega_{j} \in C_{k}$, $\int_{\omega_{j}} b \phi_{k}^{\alpha} d \mu=0$, also by (17). Consequently,

$$
\begin{aligned}
H_{k}\left(b \phi_{k}^{\alpha}\right)(x)=\frac{1}{2} \frac{1}{\mu(I)} \sum_{\omega_{j} \subset I ; \omega_{j} \in c_{k}} \int_{\omega_{j}} b(t) \phi_{k}^{\alpha_{k}}(t) \\
\times\left[\cot \left(\frac{\pi\left(x_{k}-t_{k}\right)}{p_{k}}\right)-\cot \left(\frac{\pi\left(x_{k}-t_{k}^{j}\right)}{p_{k}}\right)\right] d \mu(t),
\end{aligned}
$$

where $t^{j}=\left\{t_{k}^{j}\right\}_{k>0}$ is any fixed point in $\omega_{j}$. Thus for any coset $I$ of $G_{k}$, 


$$
\begin{aligned}
& \int_{I \cap^{c} \Omega^{*}}\left|H_{k}\left(b \phi_{k}^{\alpha}\right)(x)\right| d \mu(x) \\
& \begin{aligned}
\leqslant \frac{1}{2} \frac{1}{\mu(I)} \sum_{\omega_{j} \subset I ; \omega_{j} \in c_{k}} \int_{\omega_{j}}|b(t)| \int_{I \cap^{c} \Omega_{j}^{*}} \mid \cot \left(\frac{\pi\left(x_{k}-t_{k}\right)}{p_{k}}\right) \\
\quad-\cot \left(\frac{\pi\left(x_{k}-t_{k}^{j}\right)}{p_{k}}\right) \mid d \mu(x) d \mu(t) .
\end{aligned}
\end{aligned}
$$

A simple calculation shows that, for $t \in \omega_{j}$,

$$
\frac{1}{\mu(I)} \int_{I n^{c} \omega_{j}^{*}}\left|\cot \left(\frac{\pi\left(x_{k}-t_{k}\right)}{p_{k}}\right)-\cot \left(\frac{\pi\left(x_{k}-t_{k}^{j}\right)}{p_{k}}\right)\right| d \mu(x) \leqslant C,
$$

so we obtain

$$
\int_{I \cap c_{\Omega^{*}}}\left|H_{k}\left(b \phi_{k}^{\alpha}\right)\right| d \mu \leqslant C \sum_{\omega_{j} \subset I ; \omega_{j} \in c_{k}} \int_{\omega_{j}}|b| d \mu \leqslant C \sum_{\omega_{j} \subset I ; \omega_{j} \in c_{k}} \int_{\omega_{j}}|f| d \mu,
$$

by (18). Therefore

$$
\begin{aligned}
\mu\left\{x \notin \Omega^{*}:\right. & \left.\sum_{k=0}^{\infty}\left|H_{k}\left(b \phi_{k}^{\alpha}\right)(x)\right|>y / 4\right\} \\
& \leqslant C y^{-1} \sum_{k=0}^{\infty} \int_{c_{\Omega^{*}}}\left|H_{k}\left(b \phi_{k}^{\alpha_{k}}\right)\right| d \mu \leqslant C y^{-1} \sum_{k=0}^{\infty} \sum_{\omega_{j} \in c_{k}} \int_{\omega_{j}}|f| d \mu \\
& =C y^{-1} \sum_{j} \int_{\omega_{j}}|f| d \mu \leqslant C y^{-1}\|f\|_{1} .
\end{aligned}
$$

This establishes (31), and hence completes the proof of Theorem $1^{*}$.

\section{REFERENCES}

1. A. P. Calderón and A. Zygmund, On the existence of certain singular integrals, Acta Math. 88 (1952), 85-139. MR 14, 637.

2. J. A. Gosselin, Almost everywhere convergence of Vilenkin-Fourier series, Trans. Amer. Math. Soc. 185 (1973), 345-370.

3. C. W. Onneweer, On moduli of continuity and divergence of Fourier-series on groups, Proc. Amer. Math. Soc. 29 (1971), 109-112. MR 44 \#456.

4. - Absolute convergence of Fourier series on certain groups, Duke Math. J. 39 (1972), 599-609. MR 47 \#5524.

5. C. W. Onneweer and D. Waterman, Uniform convergence of Fourier series on groups. 1, Michigan Math. J. 18 (1971), 265-273. MR 45 \#4063.

6. R. E. A. C. Paley, A remarkable series of orthogonal functions. I, Proc. London Math. Soc. 34 (1932), 241-264.

7. J. J. Price, Certain groups of orthonormal step functions, Canad. J. Math. 9 (1957), 413-425. MR 19, 411.

8. N. Ja. Vilenkin, On a class of complete orthonormal systems, Izv. Akad. Nauk SSSR Ser. Mat. 11 (1947), 363-400; English transl., Amer. Math. Soc. Transl. (2) 28 (1963), 135. MR 9, 224; 27 \#001. 
9. C. Watari, On generalized Walsh Fourier series, Tohoku Math. J. (2) 10 (1958), 211-241. MR 21 \#1478.

10. A. Zygmund, Trigonometric series. Vols. I, II, 2nd rev. ed., Cambridge Univ. Press, New York, 1968. 38 \#4882.

DEPARTMENT OF MATHEMATICS, NORTHWESTERN UNIVERSITY, EVANSTON, ILLINOIS 60201

Current address: Department of Mathematics, University of Chicago, Chicago, Illinois 60637 